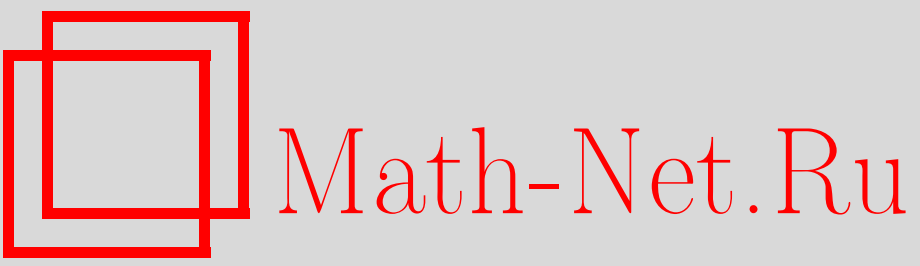

Л. М. Сладь, Спектры масс в теории бесконечнокомпонентных полей с двойной симметрией, ТМФ, 2005, том 142, номер 1, 21-36

DOI: https://doi.org/10.4213/tmf1766

Использование Общероссийского математического портала Math-Net.Ru подразумевает, что вы прочитали и согласны с пользовательским соглашением

http://www.mathnet.ru/rus/agreement

Параметры загрузки:

IP: 44.207 .124 .84

26 апреля 2023 г., 15:30:01 


\section{СПЕКТРЫ МАСС В ТЕОРИИ БЕСКОНЕЧНОКОМПОНЕНТНЫХ ПОЛЕЙ С ДВОЙНОЙ СИММЕТРИЕЙ}

Рассматривается задача о характеристиках спектров масс в дважды симметричной теории полей, которые преобразуются по представлениям собственной группы Лоренца, разложимым в бесконечную прямую сумму конечномерных неприводимых представлений. Показано, что существует такая область свободных параметров теории, в которой спектры масс фермионов являются вполне удовлетворительными с физической точки зрения и соответствуют картине, ожидаемой в партонной модели адронов.

Ключевые слова: релятивистски-инвариантные теории, бесконечнокомпонентные поля, двойные симметрии, спектры масс.

\section{1. ВВЕДЕНИЕ}

В работах [1], [2] было положено начало исследованиям релятивистски-инвариантной теории полей, которые преобразуются по представлениям собственной группы Лоренца $L_{+}^{\uparrow}$, разложимым в бесконечную прямую сумму конечномерных неприводимых представлений (такие поля мы относим к классу ISFIR).

Структура релятивистски-инвариантных лагранжианов любых свободных полей, имеющих вид

$$
\mathcal{L}_{0}=\frac{i}{2}\left[\left(\Psi, \Gamma^{\mu} \partial_{\mu} \Psi\right)-\left(\partial_{\mu} \Psi, \Gamma^{\mu} \Psi\right)\right]-(\Psi, R \Psi),
$$

найдена и описана Гельфандом и Ягломом [3], [4]. В случае полей класса ISFIR матричные операторы $\Gamma^{\mu}$ из лагранжиана (1) содержат в себе бесконечное количество произвольных констант. Из-за этого в работе [1] проводится сужение теории полей класса ISFIR, получаемое в результате наложения требования инвариантности лагранжиана (1) относительно следующих преобразований вторичной симметрии ${ }^{1)}$ :

$$
\Psi^{\prime}(x)=e^{-i D^{\mu} \theta_{\mu}} \Psi(x) .
$$

\footnotetext{
1) Понятие двойной симметрии, состоящей из первичной и вторичной симметрии, было введено в работе [5]. Оно может рассматриваться как обобщение суперсимметрии и симметрии $\sigma$-модели Гелл-Манна-Леви [6].
}

* Научно-исследовательский институт ядерной физики им. Д. В. Скобельцына, Москва, Россия. E-mail: slad@theory.sinp.msu.ru 
В формуле (2) параметры $\theta_{\mu}$ являются полярными или аксиальными 4-векторами ортохронной группы Лоренца $L^{\uparrow}$, а операторы $D^{\mu}$ имеют матричную реализацию. Указанное требование приводит к отбору счетного множества вариантов теории, каждый из которых характеризуется вполне определенным представлением групшы $L_{+}^{\uparrow}$, однозначными (с точностью до нормировочной константы) 4-векторными операторами $\Gamma^{\mu}$ и $D^{\mu}$ и тем, что оператор $R$ кратен единичному оператору $E$.

Чтобы выполненное в [1] расширение группы Лоренца не приводило в спектре масс к бесконечному вырождению по спину, ожидаемому в соответствии с теоремой Коулмена-Мандулы [7], в работе [2] постулируется спонтанное нарушение вторичной симметрии, а именно предполагается, что скалярные (относительно группы $L^{\uparrow}$ ) компоненты одного или нескольких бозонных бесконечнокомпонентных полей класса ISFIR имеют ненулевые вакуумные средние $\lambda^{i}$ (индекс $i$ может, например, обозначать ту или иную компоненту некоторого представления группы внутренней симметрии $S U(3))$. Тогда, полагая, что оператор $R$, задающий массовый член в лагранжиане свободного фермионного поля, полностью обусловлен спонтанным нарушением вторичной симметрии, имеem

$$
R=\sum_{i} \lambda^{i} Q_{i}^{(0,1) 00}
$$

Операторы $Q_{i}^{(0,1) 00}$ приходят в соотношение (3) из лагранжиана фермион-бозонного взаимодействия

$$
\mathcal{L}_{\mathrm{int}}=\sum_{i^{\prime}, \tau, l, m}\left(\psi(x), Q_{i^{\prime}}^{\tau l m} \varphi_{\tau l m}^{i^{\prime}}(x) \psi(x)\right),
$$

где $\psi(x)$ - фермионное поле, $\varphi_{\tau l m}^{i^{\prime}}(x)$ - компонента бозонного поля, характеризуемая конечномерным неприводимым представлением $\tau=\left(l_{0}, l_{1}\right)$ группы $L_{+}^{\uparrow}$, спином $l \quad(l=$ $\left.\left|l_{0}\right|,\left|l_{0}\right|+1, \ldots,\left|l_{1}\right|-1\right)$ и его проекцией на третью ось $m$, а $Q_{i^{\prime}}^{\tau l m} \equiv Q_{i^{\prime}}^{\left(l_{0}, l_{1}\right) l m}$ - матричные операторы. Индекс $i^{\prime}$ в формуле (4) может включать в себя некоторый индекс $i$ из соотношения (3) и, вдобавок к этому, сведения о трансформационных свойствах соответствуюшего бозонного поля относительно пространственного отражения. Решение вопроса о существовании нетривиального дважды симметричного лагранжиана (4) и о матричных элементах оператора $Q_{i}^{(0,1) 00}$ дано в [2].

Разделяя неоднократно высказывавшуюся веру [8] в то, что монолокальные бесконечнокомпонентные поля могут быть эффективными образами составных частиц, и используя результаты работ [1], [2], мы приступаем к изучению возможности описания адронов и их взаимодействий с помощью дважды симметричной теории полей класса ISFIR. Чтобы такая возможность была сколь-нибудь реальной, рассматриваемая теория должна удовлетворять по крайней мере следуюшим двум требованиям. Во-первых, она должна обеспечивать существование физически приемлемых спектров масс. Во-вторых, упругое электромагнитное взаимодействие основного фермионного состояния теории должно описываться формфакторами, похожими на нуклонные. Первое из этих требований рассматривается в настоящей статье.

Напомним, что именно спектры масс были камнем преткновения для всех ранее предпринимавшихся попыток последовательного релятивистского описания частиц с беско- 
нечным числом степеней свободы, реализуемых в виде различных состояний одного и того же поля, так как они во всех рассмотренных случаях имели точку сгушения, расположенную в нуле. К такому заключению относительно спектров масс пришли, в частности, Гинзбург и Тамм [9], Юкава [10], Широков [11], Марков [12] в своих анализах отдельных билокальных уравнений, а также Гельфанд и Яглом [3], Комар и др. [13] по отношению к общим линейным релятивистски-инвариантным уравнениям, основанным на представлениях группы $L_{+}^{\uparrow}$, разложимых в конечную прямую сумму бесконечномерных неприводимых представлений.

Тем более значимым представляется полученный в настояшей работе результат: дважды симметричной теории бесконечнокомпонентных полей класса ISFIR присущи спектры масс, в качественном плане воспроизводящие характерные черты экспериментальной физики адронов и партонной модели с мешками. Его обоснование и развернутая формулировка дается только для фермионных полей в вариантах теории с одним свободным параметром. Варианты теории с тремя, пятью и т.д. свободными параметрами при достаточно высоких положениях уровней спектров масс (относительно основного уровня) с любой заданной точностью сводятся к вариантам с одним параметром. Некоторые из полученных нами характеристик спектров масс предстают как следствие точных аналитических выкладок, а другие - как итог применения численных методов.

После решения основной задачи некоторое внимание в статье уделяется вопросу сопоставления теоретического спектра масс с уровнями адронных резонансов. При этом отмечается нетривиальная особенность полей класса ISFIR, заключающаяся в том, что одно состояние такого поля может иметь экспериментальное проявление в виде группы из нескольких близколежащих резонансов с различными спинами. Указывается возможная конкретизация таких групп для случая нуклонных резонансов (см. ниже таблицу). Уровень достоверности этой конкретизации не может быть установлен иначе, чем с помощью обширного анализа, включающего в себя, во-первых, предполагаемое соответствие между теоретическими и экспериментальными нуклонными и $\pi$-мезонными уровнями, во-вторых, нахождение волновых функций каждого из вовлеченных в анализ теоретических состояний, в-третьих, сравнение результатов теоретического (на основании лагранжиана (3)) и экспериментального парциально-волнового анализа амплитуд рождения $\pi N$-состояний в областях наблюдаемых нуклонных резонансов.

\section{2. УРАВНЕНИЯ И УСЛОВИЯ ДЛЯ ВЕКТОРОВ СОСТОЯНИЯ ЧАСТИЦ, ОПИСЫВАЕМЫХ ПОЛЯМИ КЛАССА ISFIR}

Предметом нашего анализа являются линейные релятивистски-инвариантные уравнения для свободного фермионного поля класса ISFIR

$$
\left(\Gamma^{\mu} \partial_{\mu}+i R\right) \psi(x)=0
$$

соответствующие лагранжианам (1).

Для поля, отвечающего плоской волне с нулевым пространственным волновым вектором $\psi(x)=e^{-i M t} \psi_{M 0}$, уравнение (5) сводится к виду

$$
\left(M \Gamma^{0}-R\right) \psi_{M 0}=0 .
$$


Это уравнение при каждом допустимом значении спина и его третьей проекции представляет собою одно или несколько рекуррентных соотношений относительно компонент бесконечнокомпонентного поля $\psi_{M 0}$. Эти соотношения сами по себе при любом значении величины $M$ дают с точностью до числового множителя одно или несколько линейно независимых решений.

Если бы уравнение (6) являло собой замкнутую математическую задачу, то оно, скорее всего, дополнялось бы условием нормируемости своих решений. Однако в физике частиц нас интересуют не столько поля, сколько амплитуды процессов, которые в рассматриваемой теории выражаются через токи вида $\left(\psi_{M 0}, O \psi_{M p}\right)$. Вектор поля $\psi_{M p}$ получается из вектора $\psi_{M 0}$ в результате такого перехода от одной системы отсчета к другой, при котором волновой 4-вектор $\{M, 0,0,0\}$ преобразуется в 4-вектор $\{E, 0,0, p\}$. В качестве оператора $O$ может выступать любой из матричных операторов теории: $Q_{i^{\prime}}^{\tau l m}, \Gamma^{\mu}, D^{\mu}$ или $R$. Токи $\left(\psi_{M 0}, O \psi_{M p}\right)$, в свою очередь, выражаются через бесконечные ряды, члены которых квадратичны по компонентам векторов $\psi_{M 0}$ и линейны по матричным элементам конечных преобразований собственной группы Лоренца. В связи с этим вместо условия нормируемости решений уравнения (6) мы вводим более жесткое требование сходимости возникающих рядов, которое мы будем называть условием конечности амплитуд. Матричные элементы любого из названных операторов $O$, связывающие неприводимые представления $\tau=\left( \pm 1 / 2, l_{1}\right)$ и $\tau^{\prime}=\left(1 / 2, l_{1}+n^{\prime}\right)$ или $\tau^{\prime \prime}=\left(-1 / 2, l_{1}+n^{\prime \prime}\right)$, где $n^{\prime}$ и $n^{\prime \prime}$ - целые числа, имеют при $l_{1} \rightarrow+\infty$ асимптотическое поведение вида $l_{1}^{\beta}$, где $\beta$ - некоторая константа. Учитывая это и асимптотику при $l_{1} \rightarrow+\infty$ компонент векторов поля $\psi_{M 0}$ и $\psi_{M p}$, которая приводится в настоящей работе, можно убедиться в том, что при любом фиксированном значении $M$ или все ряды, соответствующие перечисленным токам, сходятся, или все расходятся. Поэтому условие конечности амплитуд мы формулируем для любого значения $p$ в виде соотношения

$$
\left|\left(\psi_{M 0}, R \psi_{M p}\right)\right|<+\infty
$$

или

$$
\left(\psi_{M^{\prime} 0}, R \psi_{M p}\right)=a(p) \delta\left(M^{\prime}-M\right),
$$

где $a(p)$ - некоторое ненулевое число. Те и только те векторы поля $\psi_{M 0}$, которые удовлетворяют уравнению (6) и соотношению (7) или (8), мы будем назьвать векторами состояния частицы с массой $M$, являюшейся точкой соответственно дискретной или непрерывной части спектра масс.

Используя нижеследующую формулу (19) и подходящий аналог формулы (18), нетрудно убедиться в том, что если соотношение (8) выполняется при некотором значении $p=p_{0}$, то оно не может вьполняться при $p>p_{0}$. Таким образом, в рассматриваемой теории полей класса ISFIR спектр масс не может иметь непрерывной части.

Условие конечности амплитуд (7) или (8) при $p=0$ преврашается в условие нормируемости решений уравнения (6). Отметим здесь, что условие конечности амплитуд и условие нормируемости решений приводят к одинаковым спектрам масс во всех вариантах теории, рассматриваемых далее в разделах 4-6. В некоторых вариантах, рассматриваемых в разделе 3 , имеется непустой дискретный спектр масс при выполнении условия 
нормируемости решений уравнения (6), в то время как требование конечности амплитуд приводит к пустому спектру масс.

Приведем нужные нам результаты из работ [1] и [2], относяшиеся к рассматриваемой дважды симметричной теории полей класса ISFIR со спонтанно нарушенной вторичной симметрией.

Представление $S$ группы $L_{+}^{\uparrow}$, по которому преобразуется поле, должно совпадать с одним из бесконечномерных представлений $S^{k_{1}}\left(k_{1}\right.$ - полуцелое число, $\left.k_{1} \geqslant 3 / 2\right)$ :

$$
S^{k_{1}}=\sum_{n_{1}=0}^{+\infty} \sum_{n_{0}=-k_{1}+1 / 2}^{k_{1}-3 / 2} \oplus\left(\frac{1}{2}+n_{0}, k_{1}+n_{1}\right) .
$$

В пространстве представления $S^{k_{1}}$ оператор $\Gamma^{0}$ задается соотношениями

$$
\begin{aligned}
\Gamma^{0} \xi_{\left(l_{0}, l_{1}\right) l m}= & c_{0} D\left(l_{1}\right) \sqrt{\frac{\left(l-l_{0}\right)\left(l+l_{0}+1\right)\left(k_{1}-l_{0}-1\right)\left(k_{1}+l_{0}\right)}{\left(l_{1}-l_{0}\right)\left(l_{1}-l_{0}-1\right)\left(l_{1}+l_{0}\right)\left(l_{1}+l_{0}+1\right)}} \xi_{\left(l_{0}+1, l_{1}\right) l m}+ \\
& +c_{0} D\left(l_{1}\right) \sqrt{\frac{\left(l-l_{0}+1\right)\left(l+l_{0}\right)\left(k_{1}-l_{0}\right)\left(k_{1}+l_{0}-1\right)}{\left(l_{1}-l_{0}+1\right)\left(l_{1}-l_{0}\right)\left(l_{1}+l_{0}-1\right)\left(l_{1}+l_{0}\right)}} \xi_{\left(l_{0}-1, l_{1}\right) l m}- \\
& -c_{0} D\left(l_{0}\right) \sqrt{\frac{\left(l_{1}-l\right)\left(l_{1}+l+1\right)\left(l_{1}-k_{1}+1\right)\left(l_{1}+k_{1}\right)}{\left(l_{1}-l_{0}\right)\left(l_{1}-l_{0}+1\right)\left(l_{1}+l_{0}\right)\left(l_{1}+l_{0}+1\right)}} \xi_{\left(l_{0}, l_{1}+1\right) l m}- \\
& -c_{0} D\left(l_{0}\right) \sqrt{\frac{\left(l_{1}-l-1\right)\left(l_{1}+l\right)\left(l_{1}-k_{1}\right)\left(l_{1}+k_{1}-1\right)}{\left(l_{1}-l_{0}-1\right)\left(l_{1}-l_{0}\right)\left(l_{1}+l_{0}-1\right)\left(l_{1}+l_{0}\right)}} \xi_{\left(l_{0}, l_{1}-1\right) l m},
\end{aligned}
$$

где $\xi_{\left(l_{0}, l_{1}\right) l m}$ - вектор канонического базиса в пространстве неприводимого представления $\tau=\left(l_{0}, l_{1}\right) \in S^{k_{1}}$, а $c_{0}$ - произвольная действительная константа. Функция $D(j)$ от полуцелого аргумента $j$ описывается формулой

$$
D(j)=1
$$

если вторичная симметрия теории порождается полярным 4-векторным представлением группы $L^{\uparrow}$ (следствие 1 работы [1]), и формулой

$$
D(j)=(-1)^{j-1 / 2} j,
$$

если вторичная симметрия теории порождается аксиальным 4-векторным представлением группы $L^{\uparrow}$ (следствие 2 (п. 2) работы [1]).

Для оператора $R$ в пространстве представления $S^{k_{1}}$ для обоих рассматриваемых вариантов вторичной симметрии теории справедливы одни и те же соотношения:

$$
\begin{gathered}
R \xi_{\left(l_{0}, l_{1}\right) l m}=r\left(l_{0}, l_{1}\right) \xi_{\left(l_{0}, l_{1}\right) l m}=\left[\sum_{i} \lambda^{i} q_{i}\left(l_{0}, l_{1}\right)\right] \xi_{\left(l_{0}, l_{1}\right) l m}, \\
q_{i}\left(-l_{0}, l_{1}\right)=q_{i}\left(l_{0}, l_{1}\right), \\
\left(k_{1}-l_{0}-1\right)\left(k_{1}+l_{0}\right) q_{i}\left(l_{0}+1, l_{1}\right)+\left(k_{1}-l_{0}\right)\left(k_{1}+l_{0}-1\right) q_{i}\left(l_{0}-1, l_{1}\right)- \\
-\left(k_{1}-l_{1}-1\right)\left(k_{1}+l_{1}\right) q_{i}\left(l_{0}, l_{1}+1\right)-\left(k_{1}-l_{1}\right)\left(k_{1}+l_{1}-1\right) q_{i}\left(l_{0}, l_{1}-1\right)= \\
=z_{i}\left(l_{1}-l_{0}\right)\left(l_{1}+l_{0}\right) q_{i}\left(l_{0}, l_{1}\right),
\end{gathered}
$$


где $z_{i}=2-H_{i}^{\mathrm{B}} / H^{\mathrm{F}}$. Величины $H_{i}^{\mathrm{B}}$ и $H^{\mathrm{F}}$ - это нормировочные константы векторных операторов $D^{\mu}\left(D^{\mu} D_{\mu}=H E\right)$, входящих в преобразования (2) бозонного $\varphi^{i}(x)$ и фермионного $\psi(x)$ полей, соответственно. Они независимы друг от друга. При некотором фиксированном значении $H^{\mathrm{F}}$ величина $H_{i}^{\mathrm{B}}$ и тем самым параметр $z_{i}$ могут принимать любые значения. В самом деле, если преобразование вторичной симметрии (2) бозонного поля $\varphi^{i}(x)$ нетривиально $\left(D^{\mu} \neq 0\right)$ и это поле комплексно (а именно такие поля рассматривались в работах [1] и [2]), то $H_{i}^{\mathrm{B}}>0$. Если преобразование (2) нетривиально, а бозонное поле действительно, то $H_{i}^{\mathrm{B}}<0$. Если же преобразование $(2)$ бозонного поля тривиально, то $z_{i}=2$, оператор $Q_{i}^{(0,1) 00}$ кратен единичному оператору $E$, а наличие у этого поля ненулевого вакуумного среднего не влияет на вторичную симметрию.

В дальнейшем мы будем иметь дело только с фермионными полями, преобразующимися по "низшему" из представлений $S^{k_{1}}(9)$ собственной группы Лоренца, а именно по представлению $S^{3 / 2}$, в разложение которого входят все те и только те конечномерные неприводимые представления, которые содержат спин $1 / 2$. Решение рекуррентого соотношения (15) для этого представления $\left(k_{1}=3 / 2\right)$ было дано в работе [2] через полиномы Гегенбауэра и через гипергеометрические ряды. Оказывается, что это решение можно выразить и через элементарные функции:

$$
q_{i}\left(-\frac{1}{2}, l_{1}\right)=q_{i}\left(\frac{1}{2}, l_{1}\right)=2 q_{i 0} \frac{u_{i}^{N}\left(u_{i} N+N+1\right)-w_{i}^{N}\left(w_{i} N+N+1\right)}{N(N+1)\left(u_{i}-w_{i}\right)\left(2+u_{i}+w_{i}\right)}
$$

где $N=l_{1}-1 / 2, u_{i}=\left(z_{i}+\sqrt{z_{i}^{2}-4}\right) / 2$ и $w_{i}=\left(z_{i}-\sqrt{z_{i}^{2}-4}\right) / 2$.

В соответствии с формулами (10) и (13) операторы $\Gamma^{0}$ и $R$ диагональны по спиновому индексу $l$ и по индексу проекции спина $m$, причем их матричные элементы не зависят от $m$. Поэтому каждому вектору $\psi_{M 0}$, удовлетворяющему уравнению (6), можно поставить в соответствие определенные значения спина и его проекции. Компоненты этого вектора можно взять не зависящими от величины $m$. Линейно независимые решения уравнения (6) можно выбрать так, чтобы они обладали определенной $P$-четностью. Напомним, что $P \xi_{\left( \pm 1 / 2, l_{1}\right) l m}=(-1)^{l-1 / 2} \xi_{\left(\mp 1 / 2, l_{1}\right) l m}$. Если некоторьй вектор $\sum_{l_{1}}\left[\chi\left(l_{1}\right) \xi_{\left(-1 / 2, l_{1}\right) l m}+\chi\left(l_{1}\right) \xi_{\left(1 / 2, l_{1}\right) l m}\right]$, обладающий $P$-четностью $(-1)^{l-1 / 2}$, удовлетворяет уравнению (6) при $M=M_{0}$ и условию (7), то вектор $\sum_{l_{1}}\left[-\chi\left(l_{1}\right) \xi_{\left(-1 / 2, l_{1}\right) l m}+\chi\left(l_{1}\right) \xi_{\left(1 / 2, l_{1}\right) l m}\right]$, имеющий $P$-четность $(-1)^{l+1 / 2}$, тоже удовлетворяет условию (7) и уравнению (6), но при $M=-M_{0}$.

В пространстве представления $S^{3 / 2}$ группы $L_{+}^{\uparrow}$ уравнение (6) относительно компонент $\left(\psi_{M 0}\right)_{\left( \pm 1 / 2, l_{1}\right) l m} \equiv \chi_{l m}\left(l_{1}\right)$ вектора поля, имеюшего пространственную четность $(-1)^{l-1 / 2}$, принимает вид

$$
\begin{aligned}
& D\left(\frac{1}{2}\right) \frac{\sqrt{\left(l_{1}-l\right)\left(l_{1}+l+1\right)}}{2 l_{1}+1} \chi_{l m}\left(l_{1}+1\right)+D\left(\frac{1}{2}\right) \frac{\sqrt{\left(l_{1}-l-1\right)\left(l_{1}+l\right)}}{2 l_{1}-1} \chi_{l m}\left(l_{1}-1\right)- \\
& \quad-\left[\frac{D\left(l_{1}\right)(2 l+1)}{4 l_{1}^{2}-1}-\frac{1}{2 M c_{0}} r\left(l_{1}\right)\right] \chi_{l m}\left(l_{1}\right)=0
\end{aligned}
$$

где $l_{1} \geqslant l, r\left(l_{1}\right) \equiv r\left( \pm 1 / 2, l_{1}\right)$. 
Запишем релятивистски-инвариантную билинейную форму из соотношения (7) через компоненты векторов $\chi_{l m}\left(l_{1}\right)$ и через матричные элементы конечных преобразований собственной группы Лоренца

$$
\begin{aligned}
\left(\psi_{M 0}, R \psi_{M p}\right)= & \sum_{l_{0}=-1 / 2}^{1 / 2} \sum_{l_{1}=l+1}^{+\infty}(-1)^{l-1 / 2} \chi_{l m}^{*}\left(l_{1}\right) r\left(l_{1}\right) \times \\
& \times\left\{\left[e^{\alpha I^{03}}\right]_{\left(l_{0}, l_{1}\right) l m,\left(l_{0}, l_{1}\right) l m}\right\} \chi_{l m}\left(l_{1}\right),
\end{aligned}
$$

где th $\alpha=p / \sqrt{M^{2} c^{2}+p^{2}}$, a $I^{03}$ - инфинитезимальный оператор группы $L_{+}^{\uparrow}$.

Явный вид матричных элементов конечных преобразований группы $L_{+}^{\uparrow}$ для бесконечномерных унитарных неприводимых представлений, обозначаемых парой чисел $\left(l_{0}, \nu\right)$, найден в работе [14]. Рассуждения и результаты этой работы имеют силу и для интересующих нас конечномерных неприводимых представлений. Чтобы сохранить точное содержание используемых нами обозначений, нужно положить $\nu=i l_{1}$. При рассмотрении вопроса о сходимости ряда вида (18) нам достаточно знать асимптотическое поведение интересующих нас матричных элементов при $l_{1} \rightarrow+\infty$. Имеем

$$
\left[e^{\alpha I^{03}}\right]_{\left(l_{0}, l_{1}\right) l m,\left(l_{0}, l_{1}\right) l m}=T_{0} \frac{e^{\alpha l_{1}}}{l_{1}}\left(1+O\left(l_{1}^{-1}\right)\right),
$$

где величина $T_{0}$ не зависит от числа $l_{1}$.

Ниже мы обсуждаем спектры масс в двух вариантах спонтанного нарушения вторичной симметрии: нарушение вызывается одним бозонным полем класса ISFIR (в этом случае индекс $i$ будет повсеместно опускаться) или двумя бозонными полями. В первом варианте мы рассматриваем по отдельности три существенно разные области значений параметра $z:(-\infty,-2],(-2,2)$ и $(2,+\infty)$. Во втором варианте внимание уделяется только области $z_{1} \in(2,+\infty), z_{2} \in(-2,2)$.

\section{3. ПУСТОЙ СПЕКТР МАСС В ОБЛАСТИ ПАРАМЕТРА $z \in(-2,2)$}

В ситуации, когда вторичная симметрия теории нарушена спонтанно, найти решения уравнения (17) в виде элементарных или специальных функций, конечных или бесконечных рядов не удается. Не удается найти и аналитические формулы для спектров масс теории. Однако мы в состоянии получить ряд заключений относительно спектров масс, основываясь на асимптотическом поведении некоторых величин.

Пусть $z \in(-2,2)$. Тогда, используя формулу $(16)$, имеем при $l_{1} \rightarrow+\infty$

$$
r\left(l_{1}\right)=r_{0} \frac{\sin \zeta l_{1}}{l_{1}}\left(1+O\left(l_{1}^{-1}\right)\right)
$$

где $\zeta \in(0, \pi)$, а $r_{0}$ - константа. Отсюда и из уравнения (17) получаем при $l_{1} \rightarrow+\infty$

$$
\chi_{l m}\left(l_{1}\right)=A_{0}(-1)^{\left[l_{1} / 2\right]} l_{1}^{s}\left(1+K\left(l_{1}\right)+O\left(l_{1}^{-2}\right)\right)+B_{0}(-1)^{\left[\left(l_{1}+1\right) / 2\right]} l_{1}^{-s}\left(1-K\left(l_{1}\right)+O\left(l_{1}^{-2}\right)\right),
$$


где

$$
s=(2 l+1)\left(1-D\left(\frac{1}{2}\right)\right), \quad K\left(l_{1}\right)=(-1)^{l_{1}+1 / 2} \frac{r_{0}}{2 M c_{0} D(1 / 2) \sin \zeta} \cdot \frac{\cos \zeta l_{1}}{l_{1}}
$$

$[a]$ - целая часть числа $a$, а величины $A_{0}$ и $B_{0}$ не зависят от $l_{1}$.

Основываясь на этой асимптотике, нетрудно установить, что при $\alpha \neq 0$ члены ряда (18) растут при $l_{1} \rightarrow+\infty$ независимо от того, равна ли величина $A_{0}$ нулю или нет при тех или иных значениях $M$. Следовательно, если $z \in(-2,2)$, то ни при каких значениях величины $M$ решение уравнения (17) не может удовлетворять условию конечности амплитуд (7), т.е. спектр масс пуст.

\section{4. ХАРАКТЕРИСТИКИ СПЕКТРОВ МАСС В ОБЛАСТИ ПАРАМЕТРА $z \in(-\infty,-2]$}

Так как в области $z \in(-\infty,-2)$ справедливы неравенства $w<-1$ и $-1<u<0$, то из равенства (16) и уравнения (17) получаем при $l_{1} \rightarrow+\infty$

$$
\begin{gathered}
r\left(l_{1}\right)=r_{0} \frac{w^{l_{1}+1 / 2}}{l_{1}+1 / 2}\left(1+O\left(l_{1}^{-1}\right)\right), \\
\chi_{l m}\left(l_{1}\right)=A_{0} G\left(l_{1}\right)\left(1+O\left(l_{1}^{-1}\right)\right)+B_{0} G^{-1}\left(l_{1}\right)\left(1+O\left(l_{1}^{-1}\right)\right),
\end{gathered}
$$

где

$$
G\left(l_{1}\right)=\frac{v^{l_{1}-1 / 2} w^{\left(4 l_{1}^{2}-1\right) / 8}}{\left(l_{1}-1 / 2\right) !}, \quad v=-\frac{r_{0}}{M c_{0} D(1 / 2)}
$$

Если при некоторых значениях $M$ величина $A_{0}$ не равна нулю, то, очевидно, условие $(7)$ не может выполняться. Если же $A_{0}=0$ при каком-то значении $M$, то члены ряда $(18)$ при $l_{1} \rightarrow+\infty$ имеют асимптотическое поведение порядка $\left(l_{1}+1 / 2\right) !\left(l_{1}-1 / 2\right) ! \times$ $v^{-2 l_{1}} u^{\left(4 l_{1}^{2}-1\right) / 4} e^{\alpha l_{1}}$. Отношение такого члена ряда к предшествуюшему ему члену в пределе $l_{1} \rightarrow+\infty$ равно нулю. Следовательно, при обсуждаемом значении $M$ рассматриваемый ряд (18) сходится, что эквивалентно выполнению условия (7).

Итак, для всех значений параметра $z$ из области $z \in(-\infty,-2)$ и для обоих вариантов теории, выражаемых соотношениями (11) и (12), спектр масс дискретен, если он не пуст.

Аналогичное утверждение справедливо и при $z=-2$. В этом легко убедиться, приняв во внимание, что в этом случае $r\left(l_{1}\right)=r_{0}(-1)^{l_{1}+1 / 2} l_{1}$ и имеет место аналог соотношения $(24)$, в котором нужно положить $G\left(l_{1}\right)=v^{l_{1}-1 / 2}(-1)^{\left(4 l_{1}^{2}-1\right) / 8}\left(l_{1}-1 / 2\right)$ !.

Докажем теперь, что в области $z \in(-\infty,-2]$ множество всех масс теории ограничено снизу некоторым положительным числом. Для этого достаточно указать такое число $\mu_{0}>0$, что для множества значений $M$, которые удовлетворяют ограничению $|M| \leqslant$ $\mu_{0}$, компоненты поля $\chi_{l m}\left(l_{1}\right)$ не являются сколь угодно малыми при достаточно больших значениях $l_{1}$. 
Используя формулу (16), устанавливаем, что в области $z \leqslant-2$ величина $\left|r\left(l_{1}\right)\right|$ монотонно растет с ростом $l_{1}$, а функция полуцелого аргумента $r\left(l_{1}\right)$ является знакопеременной: $r\left(l_{1}+1\right) / r\left(l_{1}\right)<0$. Пусть $\mu_{1}=\left|r(3 / 2) / c_{0}\right|$ и $|M| \leqslant \mu_{1} / 2$. Тогда для обоих вариантов функции $D(j)\left((11)\right.$ и (12)) и для всех допустимых значений $l$ и $l_{1}$ величина $D\left(l_{1}\right)(l+1 / 2) /\left(l_{1}^{2}-1 / 4\right)-r\left(l_{1}\right) / M c_{0}$ по модулю больше единицы и изменяет свой знак при изменении $l_{1}$ на 1 . Это вместе с уравнением (17) дает требуемое неравенство $\left|\chi_{l m}\left(l_{1}+1\right)\right|>\left|\chi_{l m}\left(l_{1}\right)\right|$.

Для удобства мы будем в дальнейшем использовать то или иное соотношение между нормировочными константами, получая тем самым разные единицы массы. Если $r(3 / 2) /\left(2 c_{0} D(1 / 2)\right)= \pm 1$ (знаки плюс и минус относятся соответственно к соотношениям (11) и (12), они выбраны так, чтобы низшие уровни частиц со спином $1 / 2$ обладали пространственной четностью +1$)$, то введем обозначение $M_{\mathrm{c}}=|M|$. Любое количество

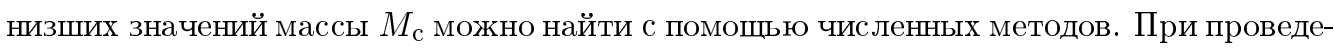
нии численных расчетов в соответствии с доказанным выше подлежит рассмотрению только область $M_{\mathrm{c}}>0.5$. Чтобы найти все точки спектра в интересуюших нас областях величины $M_{\mathrm{c}}$ и параметра $z$ при заданном спине $l$, нам достаточно ограничиться поиском таких точек $M_{\mathrm{c}}$, в которых при некотором фиксированном значении $z$ величина $A_{0}$ из формулы (24) обрашается в нуль, но не имеет минимума или максимума. Тогда, очевидно, в любых малых окрестностях указанных точек спектра масс при достаточно больших значениях $l_{1}$ величина $\chi_{l m}\left(l_{1}\right)$ изменяет свой знак. Это служит нам алгоритмом численного решения задачи о массах. Анализируя зависимость тех или иных двух соседних точек спектра масс от параметра $z$, мы можем установить, существует ли такое значение $z_{0}$, при стремлении к которому с одной из сторон эти точки становятся сколь угодно близкими друг к другу, не появляясь по другую сторону от $z_{0}$. Если такое число $z_{0}$ существует, то предельное значение двух указанных точек будет тем значением массы $M_{\mathrm{c}}$, при котором величина $A_{0}$ имеет нулевое значение и экстремум.

На рис. 1 и 2 для случаев, отвечающих соответственно соотношениям (11) и (12), изображена зависимость от параметра $z$ масс состояний со спинами $1 / 2,3 / 2,5 / 2$ и 7/2.

Отметим вначале некоторые характеристики спектра масс теории с двойной симметрией, порождаемой аксиальным 4-векторным представлением ортохронной группы Лоренца (ей отвечает соотношение (12)). Во-первых, спектр масс является непустым, если пространственная четность частиц со спином $l$ равна $(-1)^{l-1 / 2}$, и пустым, если четность равна $(-1)^{l+1 / 2}$. Во-вторых, среди массовых линий имеются такие пары, которые обрываются, сливаясь в некоторых своих обших предельных точках при $z_{0}<-2$, и имеются такие одиночные линии, которые сушествуют во всей области $z \in(-\infty,-2]$. В-третьих, для всех $z$, по крайней мере из области $(-\infty,-2.1)$, уровни спектра масс имеют одинаковое упорядочение, связанное со спином. Оно такое, как, например, при $z=-2.645$, когда низшие массы $M_{\text {с }}$ (спин и четность $l^{P}$ ) равны: $1.982(1 / 2)^{+}, 3.209(3 / 2)^{-}, 3.687(1 / 2)^{+}$, $5.470(5 / 2)^{+}, \quad 6.143(3 / 2)^{-}, \quad 6.964(1 / 2)^{+}, \quad 9.709(7 / 2)^{-}, \quad 10.72(5 / 2)^{+}$, $11.91(3 / 2)^{-}, 13.35(1 / 2)^{+}, 17.82(9 / 2)^{+}, 19.38(7 / 2)^{-}$. Массы низших уровней с тем или иным спином растут с ростом спина несколько быстрее геометрической прогрессии. Последовательность нескольких взятых подряд масс уровней с одним и тем же спином близ- 




Рис. 1. Зависимость массовых уровней от параметра $z$ при $z<-2$ в теории с двойной симметрией, порождаемой полярным 4-векторным представлением ортохронной группы Лоренца.

ка к геометрической прогрессии. Поэтому, хотя в приведенном примере отношение масс низших уровней со спином и четностью $(3 / 2)^{-}$и $(1 / 2)^{+}$равно отношению масс резонанса $N(1520)$ и нуклона, положение уровней с $l^{P}=(5 / 2)^{+},(7 / 2)^{-},(9 / 2)^{+}$и т.д. сильно отличается от положения соответствующих нуклонных резонансов [15]. В-четвертых, для значений параметра, близких к $z=-2$, упорядочение уровней спектра масс, связанное со спином, изменяется вместе с изменением $z$. Так, при $z=-2$ низшие уровни $M_{\mathrm{c}}\left(l^{P}\right)$ таковы: $9.506(5 / 2)^{+}, 12.14(9 / 2)^{+}, 12.55(3 / 2)^{-}, 13.25(5 / 2)^{+}, 15.77(13 / 2)^{+}$, $16.03(19 / 2)^{-}, 17.45(21 / 2)^{+}$.

Из характеристик спектра масс теории с двойной симметрией, порождаемой полярным 4-векторным представлением группы $L^{\uparrow}$ (ей отвечает соотношение (11)), выделим следуюшие. Во-первых, частищы при любом значении спина обладают одной и той же пространственной четностью +1 . Во-вторых, множество всех массовых линий разбивается на такие пары, которые, сливаясь, обрываются при $z_{0}<-2$. В-третьих, массы низших уровней с двумя последовательными спинами, если они существуют для данного $z$ из рассматриваемой области, отличаются друг от друга по крайней мере на порядок, что явно не соответствует картине с барионными резонансами. 


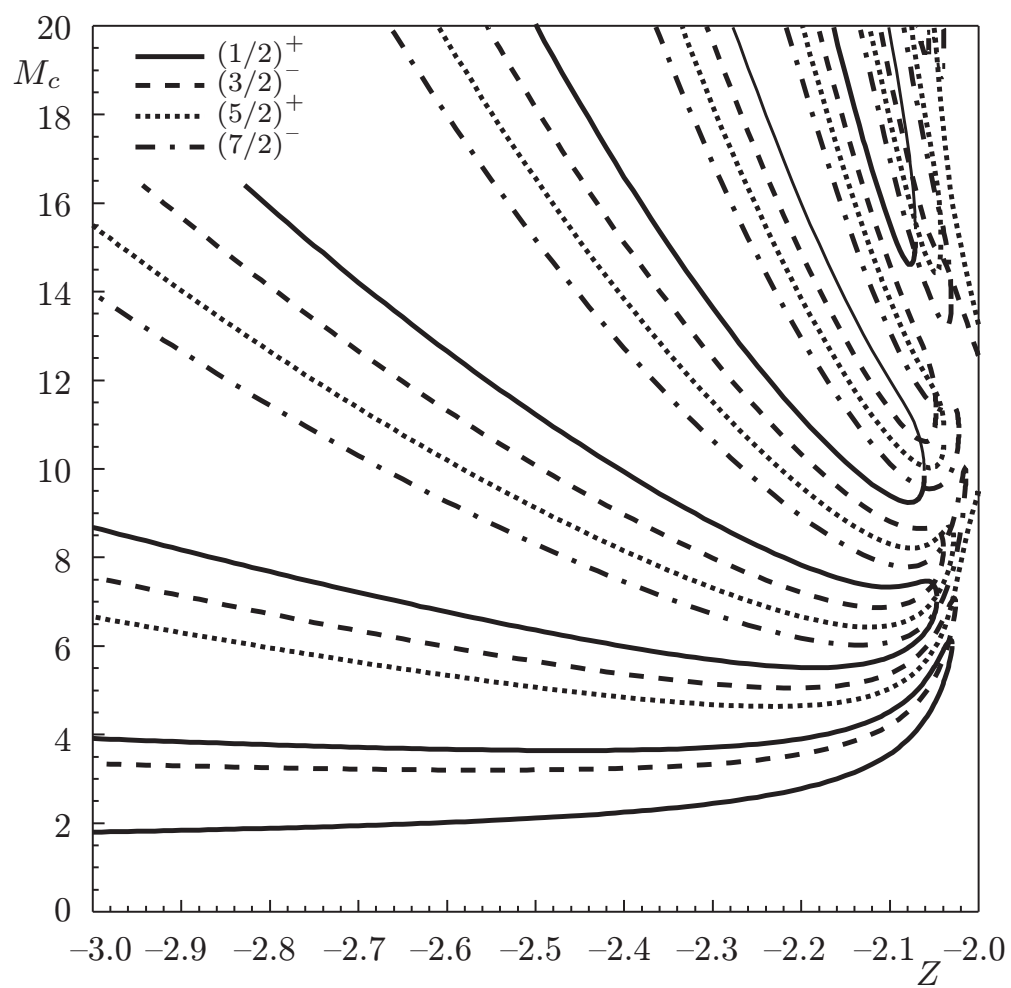

Рис. 2. Зависимость массовых уровней от параметра $z$ при $z<-2$ в теории с двойной симметрией, порождаемой аксиальным 4-векторным представлением ортохронной группы Лоренца.

\section{5. ХАРАКТЕРИСТИКИ СПЕКТРОВ МАСС В ОБЛАСТИ ПАРАМЕТРА $z \in(2,+\infty)$}

Так как в области $z \in(2,+\infty)$ выполняются неравенства $u>1$ и $0<w<1$, то справедливы формулы (23)-(25) и следующие за ними рассуждения о выполнении условия (7), если в этих формулах и рассуждениях заменить $w$ на $u$ и $u$ на $w$. Отсюда вытекает заключение, что для всех значений $z \in(2,+\infty)$ в обоих вариантах теории, отражаемых соотношениями (11) и (12), спектр масс дискретен, если он не пуст.

Из соотношения (16) получаем, что в области $z>2$ величина $r\left(l_{1}\right) / r(3 / 2)$ является положительной и монотонно растущей с ростом $l_{1}$. Этот факт и уравнение (17) при $l_{1}>l$ дают неравенство $\left|\chi_{l m}\left(l_{1}\right)\right|>\left(1+1 /\left(l_{1}-l\right)\right)\left|\chi_{l m}(l)\right|$ для всех $l$ и для всех значений $M$ из области $|M| \leqslant \mu_{1} / 3$, где $\mu_{1}=\left|r(3 / 2) / c_{0}\right|$. Следовательно, в указанной области $M$ ряд (18) расходится, условие (7) не выполняется, точки спектра масс отсутствуют, т.е. спектр масс ограничен снизу.

На рис. 3 и 4, отвечаюших формулам (11) и (12), соответственно, изображена зависимость низших уровней масс от параметра $z$ для спинов $1 / 2,3 / 2,5 / 2$ и $7 / 2$, каждому из которых сопоставляются пространственные четности +1 и -1 . В обоих вариантах тео-

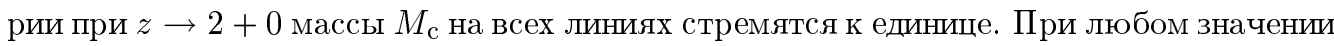


$z>2$ массы нескольких низших уровней с последовательными спинами $l$ и с четностями $(-1)^{l-1 / 2}$ образуют приближенно геометрическую прогрессию. Подобная закономерность имеет место и для масс нескольких последовательных уровней с одними и теми же спином и четностью. Из-за этого нельзя обеспечить сколь-нибудь удовлетворительного количественного согласия между низшими уровнями теории с одним параметром $z$ и уровнями нуклонных резонансов. Существенное различие двух вариантов теории состоит в порядке взаимных расположений низших уровней с двумя последовательными значениями спина и той или другой четностью. Чтобы проиллюстрировать все это, ниже будут приведены числовые примеры, в которых отношение масс низших уровней с $l^{P}=(3 / 2)^{-}$и $l^{P}=(1 / 2)^{+}$, даваемых рассматриваемой теорией, то же, что и для резонанса $N(1520)$ и нуклона.

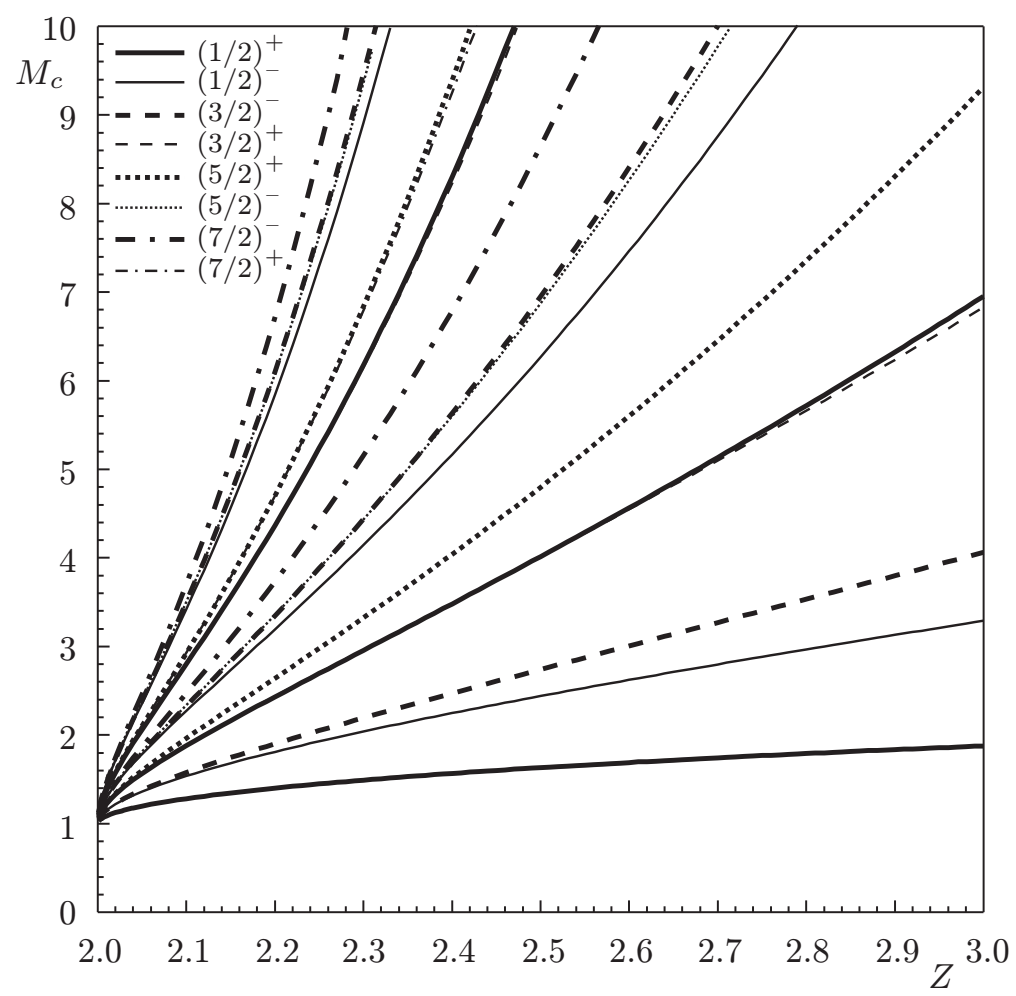

Рис.3. Зависимость массовых уровней от параметра $z$ при $z>2$ в теории с двойной симметрией, порождаемой полярным 4-векторным представлением ортохронной группы Лоренца.

Отметим несколько характеристик спектра масс для варианта теории с соотношением (11). Во-первых, упорядочение уровней масс, связанное со спином и четностью, одинаково для всех значений параметра $z$ из области $(2,+\infty)$. Оно таково, как, например,

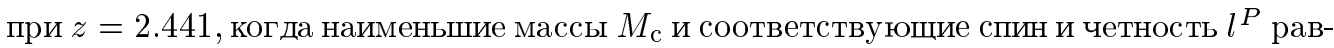
ны: $1.595(1 / 2)^{+}, 2.328(1 / 2)^{-}, 2.582(3 / 2)^{-}, 3.699(1 / 2)^{+}, 3.700(3 / 2)^{+}, 4.346(5 / 2)^{+}$, $5.609(1 / 2)^{-}, 6.111(5 / 2)^{-}, 6.158(3 / 2)^{-}, 7.522(7 / 2)^{-}, 9.272(1 / 2)^{+}, 9.174(3 / 2)^{+}$. Bo- 


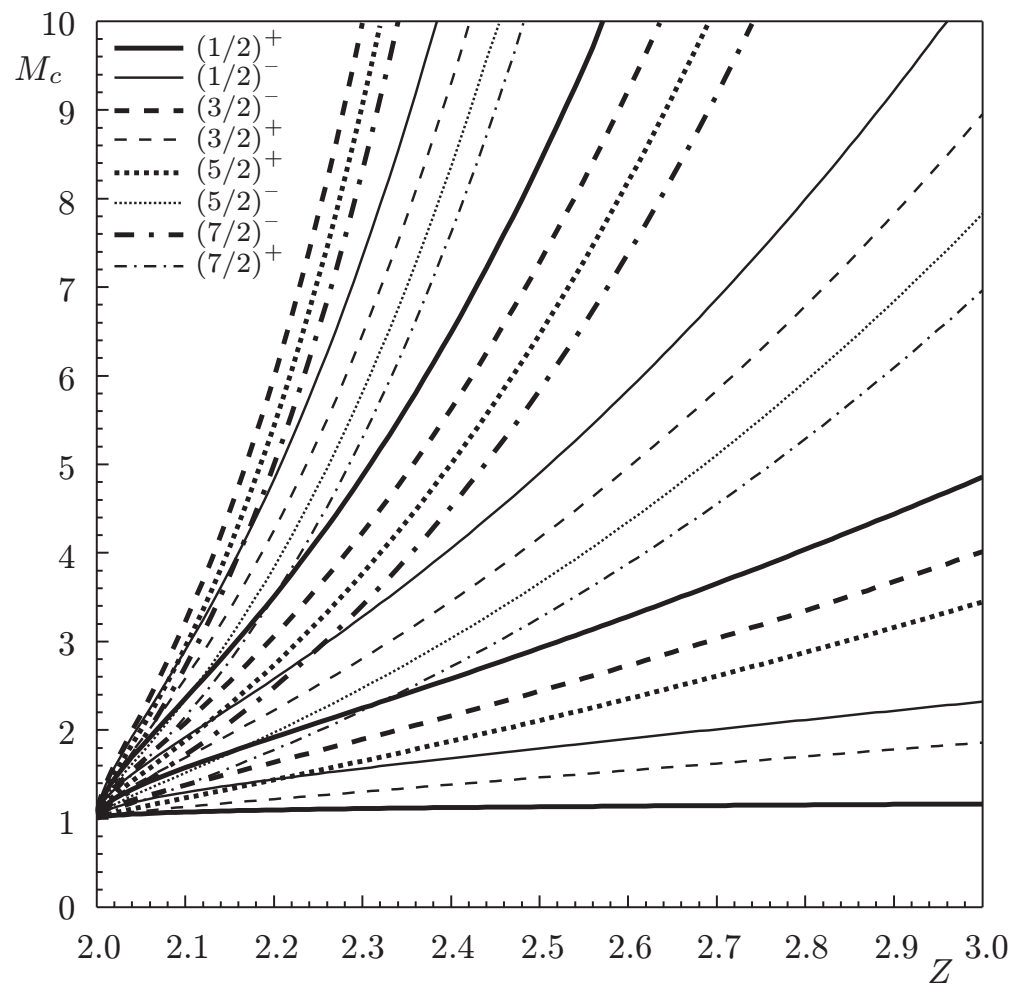

Рис.4. Зависимость массовых уровней от параметра $z$ при $z>2$ в теории с двойной симметрией, порождаемой аксиальным 4-векторным представлением ортохронной группы Лоренца.

вторых, некоторые уровни масс с различными $l^{P}$ мало отличаются друг от друга в значительной окрестности точки $z=2$. Такая малость различия имеет место для $(n+1)$-го уровня с $l^{P}=(1 / 2)^{+}$и $n$-го уровня с $l^{P}=(3 / 2)^{+}$, для $(n+1)$-го уровня с $l^{P}=(3 / 2)^{-}$ и $n$-го уровня с $l^{P}=(5 / 2)^{-}$, для $(n+1)$-го уровня с $l^{P}=(5 / 2)^{+}$и $n$-го уровня с $l^{P}=$ $(7 / 2)^{+}(n \geqslant 1)$ и т.д.

Для варианта теории с соотношением (12) обшее упорядочение уровней, связанное со спином и четностью, может быть неодинаковым для разных значений параметра $z$. Упорядочение же низших уровней с теми или иными $l^{P}$ одинаково для всех $z>2$. Это упорядочение такое, как в следуюшем примере, где приводятся все уровни с $M_{\mathrm{c}}<4$, вычисленные при $z=2.261: 1.108(1 / 2)^{+}, \quad 1.272(3 / 2)^{+}, \quad 1.521(1 / 2)^{-}, \quad 1.567(5 / 2)^{+}$, $1.794(3 / 2)^{-}, \quad 2.041(7 / 2)^{+}, \quad 2.119(1 / 2)^{+}, \quad 2.272(5 / 2)^{-}, \quad 2.573(3 / 2)^{+}, \quad 2.769(9 / 2)^{+}$, $2.999(1 / 2)^{-}, 3.027(7 / 2)^{-}, 3.342(5 / 2)^{+}, 3.747(3 / 2)^{-}, 3.876(11 / 2)^{+}$.

Итак, дважды симметричная теория с одним параметром $z$, связанным со спонтанным нарушением вторичной симметрии, в целом дает следуюшую качественную картину для спектров масс. При любых значениях $z$ не сушествует непрерывной части спектра масс. Если при некотором $z$ спектр масс не пуст, то массовые уровни ограничены снизу положительной величиной. В области параметра $z>2$ каждому значению спина и

2 Теоретическая и математическая физика, т. 142, № 1, 2005 г. 
четности соответствует счетное множество масс, простирающихся до бесконечности, а наименшшие значения уровней масс с тем или иным спином растут с ростом спина. Удовлетворительного количественного согласия с уровнями нуклонных резонансов теория с одним параметром $z$ не дает.

\section{6. СОПОСТАВЛЕНИЕ СПЕКТРА МАСС ТЕОРИИ С ДВУМЯ ПАРАМЕТРАМИ $z_{i}$ С УРОВНЯМИ НУКЛОННЫХ РЕЗОНАНСОВ}

Общая ситуация со спектрами масс теории, в которой имеется два бозонных поля класса ISFIR с ненулевыми вакуумными средними своих скалярных (относительно группы $L^{\uparrow}$ ) компонент, достаточно богата различными вариантами. Мы уделим некоторое внимание только одному варианту, который предназначается для пробного количественного сопоставления теоретического спектра масс с уровнями нуклонных резонансов и описывается ниже.

Будем рассматривать теорию с двойной симметрией, порождаемой полярным 4-векторным представлением ортохронной группы Лоренца (ей отвечает соотношение (11)). При наличии двух параметров $z_{i}$ величину $r\left(l_{1}\right)$ из уравнения (17) можно записать в виде

$$
r\left(l_{1}\right)=f_{0}\left(\frac{q_{1}\left(1 / 2, l_{1}\right)}{q_{10}}+f_{2} \frac{q_{2}\left(1 / 2, l_{1}\right)}{q_{20}}\right),
$$

где $q_{i}\left(1 / 2, l_{1}\right)$ зависит от $z_{i}$ и дается формулой $(16)$. Очевидно, что в такой теории помимо нормировочной константы $f_{0} / c_{0}$ имеются три свободных параметра: $z_{1}, z_{2}$ и $f_{2}$. Выберем следующие ограничения на них: $z_{1}>2,\left|z_{2}\right|<2,\left|f_{2}\right|<1$. В заданной области параметров с ростом $l_{1}$ величина $\left|q_{1}\left(1 / 2, l_{1}\right)\right|$ монотонно растет, а $q_{2}\left(1 / 2, l_{1}\right)$ осциллирует с уменьшаюшейся амплитудой. Величины $r\left(l_{1}\right)$ и $f_{0} q_{1}\left(1 / 2, l_{1}\right) / q_{10}$ имеют одинаковый знак при всех $l_{1}$ и могут заметно отличаться друг от друга только при малых значениях $l_{1}$. На асимптотику величин $r\left(l_{1}\right)$ и $\chi_{l m}\left(l_{1}\right)$ при $l_{1} \rightarrow+\infty$ параметр $z_{2}$ не влияет. Справедливы формулы (23)-(25), если заменить в них $w$ на $u_{1}$, и справедливы вытекающие из этих формул заключения о спектрах масс. Поэтому в рассматриваемом варианте теории с двумя параметрами $z_{1}$ и $z_{2}$ по сравнению с вариантом теории с одним параметром $z_{1}$ могут существенно изменить свое положение только уровни спектра масс с низшими значениями спина $l$.

Отметим теперь крайне важное обстоятельство, присушее рассматриваемой теории бесконечнокомпонентных полей. Пусть основному фермионному и основному бозонному уровням теории отвечают частицы $F$ со спином $1 / 2$ и $B$ со спином 0 , соответственно, и пусть в теории имеется возбужденньй фермионный уровень, которому отвечает частица $F^{*}$ со спином $l$. Рассмотрим амплитуду $\mathcal{M}$ распада $F^{*} \rightarrow F B$ в системе покоя резонанса $F^{*}$, которая описывается лагранжианом (3). Так как в этой системе отсчета частицы $F$ и $B$ имеют ненулевые скорости, то сопоставляемые им бесконечнокомпонентные поля класса ISFIR имеют ненулевые компоненты со всеми полуцелыми и со всеми целыми спинами, соответственно. Поэтому амплитуда $\mathcal{M}$ имеет ненулевые слагаемые, в которых компоненты полей $F^{*}, F$ и $B$ описываются следуюшими наборами спинов: $\left\{l_{F^{*}}, l_{F}, l_{B}\right\}$ : $\{l, l, 0\},\{l, l-1,1\},\{l, l, 1\},\{l, l+1,1\},\{l, l-2,2\}, \ldots,\{l, l+2,2\}, \ldots$ Оценить величину вклада того или иного спина $l^{\prime}$ фермиона $F$ и спина $l^{\prime \prime}$ бозона $B$ в амплитуду 


\begin{tabular}{|c|c|c|c|c|c|}
\hline \multicolumn{2}{|c|}{ Теория } & \multicolumn{4}{|c|}{ Эксперименты [15] } \\
\hline$l^{P}$ & $\begin{array}{l}\text { Macca } \\
\text { (MэB) }\end{array}$ & $l^{P}$ & Резонанс & $\begin{array}{l}\text { Macca } \\
(\text { MэB })\end{array}$ & Статус \\
\hline$(1 / 2)^{+}$ & 939 & $(1 / 2)^{+}$ & $N$ & 939 & $* * * *$ \\
\hline$(1 / 2)^{+}$ & 1481 & $(1 / 2)^{+}$ & $N(1440)$ & $1430-1470^{\text {БВ }}$ & $* * * *$ \\
\hline$(1 / 2)^{-}$ & 1487 & $(1 / 2)^{-}$ & $N(1535)$ & $1495-1515$ & $* * * *$ \\
\hline$(3 / 2)^{-}$ & 1508 & $(3 / 2)^{-}$ & $N(1520)$ & $1505-1515$ & $* * * *$ \\
\hline$(3 / 2)^{+}$ & 1661 & $\begin{array}{l}(1 / 2)^{-} \\
(5 / 2)^{-} \\
(3 / 2)^{+}\end{array}$ & $\begin{array}{l}N(1650) \\
N(1675) \\
N(1720)\end{array}$ & $\begin{array}{l}1640-1680 \\
1655-1665 \\
1650-1750\end{array}$ & $\begin{array}{l}* * * * \\
* * * * \\
* * * *\end{array}$ \\
\hline$(5 / 2)^{+}$ & 1675 & $\begin{array}{l}(5 / 2)^{+} \\
(3 / 2)^{-} \\
(1 / 2)^{+}\end{array}$ & $\begin{array}{l}N(1680) \\
N(1700) \\
N(1710)\end{array}$ & $\begin{array}{l}1665-1675 \\
1630-1730 \\
1670-1770\end{array}$ & $\begin{array}{l}* * * * \\
* * * \\
* * *\end{array}$ \\
\hline$(5 / 2)^{-}$ & 1892 & $(3 / 2)^{+}$ & $N(1900)$ & $\approx 1900^{\mathrm{EB}}$ & $* *$ \\
\hline$(1 / 2)^{-}$ & 1923 & $(7 / 2)^{+}$ & $N(1990)$ & 1870-1930 & $* *$ \\
\hline$(7 / 2)^{-}$ & 1940 & $(7 / 2)^{-}$ & $N(2190)$ & $1950-2150$ & $* * * *$ \\
\hline$(3 / 2)^{-}$ & 1995 & $\int(5 / 2)^{+}$ & $N(2000)$ & $\approx 2000^{\mathrm{БB}}$ & $* *$ \\
\hline$(1 / 2)^{+}$ & 2004 & $(3 / 2)^{-}$ & $N(2080)$ & $1980-2120$ & $* *$ \\
\hline$(5 / 2)^{+}$ & 2144 & $(1 / 2)^{+}$ & $N(2100)$ & $2080-2160$ & $*$ \\
\hline$(3 / 2)^{+}$ & 2140 & $(1 / 2)^{-}$ & $N(2090)$ & $2080-2220$ & * \\
\hline$(7 / 2)^{+}$ & 2179 & $(5 / 2)^{-}$ & $N(2200)$ & $2040-2160$ & $* *$ \\
\hline & & $(9 / 2)^{-}$ & $N(2250)$ & $2080-2200$ & $* * * *$ \\
\hline$(9 / 2)^{+}$ & 2244 & $(9 / 2)^{+}$ & $N(2220)$ & $2100-2240$ & $* * * *$ \\
\hline$(11 / 2)^{-}$ & 2547 & $(11 / 2)^{-}$ & $N(2600)$ & $2550-2750^{\text {БВ }}$ & $* * *$ \\
\hline$(13 / 2)^{+}$ & 2919 & $(13 / 2)^{+}$ & $N(2700)$ & $\approx 2700^{\mathrm{БB}}$ & * \\
\hline
\end{tabular}

$\mathcal{M}$ можно только тогда, когда известны векторы состояний частиц $F^{*}, F$ и $B$ в их системах покоя. Так как экспериментальные суждения о спине резонанса составляются на основании парциально-волнового анализа, который построен на представлениях группы трехмерных врашений, а не на представлениях групшы Лоренца, то сказанное выше может находить свое отражение в заключении экспериментатора о существовании группы из нескольких резонансов, имеющих одну и ту же массу и различающихся между собой только спином. Тем самым при сопоставлении теоретического спектра масс с экспериментальным мы должны обращать особое внимание на подобные группы резонансов и принимать решение о количестве отвечающих им теоретических уровней.

Простейшее предполагаемое соответствие между рассматриваемым вариантом теории и экспериментальной картиной нуклонных резонансов отражено в таблице. Ему от- 
вечает следующий выбор нормировочной константы и свободных параметров: $f_{0} / c_{0}=$ $-939 / 2.4686 \mathrm{MэB}, z_{1}=2.036, z_{2}=0.14, f_{2}=-0.6724$. Параметры $z_{2}$ и $f_{2}$ подбирались так, чтобы массы низших уровней с $l^{P}=(3 / 2)^{-}$и $l^{P}=(5 / 2)^{+}$равнялись соответственно 1508 и 1675 МэВ. Параметр $z_{1}=2.036$ определяет состояния частиц с большими спинами. В качестве экспериментальных масс почти всех резонансов мы берем положение полюсов. Массы Брейта-Вигнера (отмеченные в таблице верхним индексом БВ) взяты, во-первых, для тех нескольких резонансов, чье положение полюсов не приводится в работе [15], и, во-вторых, для резонанса Ропера $N(1440)$, так как в окрестности 1440 МэВ найдено два полюса, $1370-114 i$ и $1360-120 i$, параметры которых сильно отличаются от обычных $M=1470 \mathrm{MэB} \mathrm{и} Г=545 \mathrm{MэB} \mathrm{[16].}$

Пробное сопоставление спектров масс рассматриваемой теории с уровнями ненуклонных резонансов еще не проводилось. Оно требует в частности разделения $\Lambda$-резонансов на синглетные и октетные, а $\Sigma$ - и $\Xi$-резонансов на октетные и декуплетные относительно группы внутренней симметрии $S U(3)$. Для описания $\Delta$-резонансов, по-видимому, следует использовать представление $S^{5 / 2}$ группы $L_{+}^{\uparrow}$, описываемое формулой $(11)$, где $k_{1}=5 / 2$. В этом случае низший массовый уровень будет иметь спин $3 / 2$. Анализ спектров масс бозонных полей класса ISFIR в любых вариантах дважды симметричной теории станет возможным лиш после нахождения структуры лагранжиана четырехчастичного взаимодействия таких полей меж ду собой и вытекаюшего из нее следствия спонтанного нарушения вторичной симметрии.

Благодарности. Я очень признателен Э. Э. Боосу, В. И. Саврину и Н. П. Юдину за полезные обсуждения и поддержку моей работы.

\section{Список литературы}

[1] Л. М. Сладь. ТМФ. 2001. Т. 129. С. 68.

[2] Л. М. Сладь. ТМФ. 2002. Т. 133. С. 54.

[3] И. М. Гельфанд, А. М. Яглом. ЖЭТТ. 1948. Т. 18. С. 703.

[4] И. М. Гельфанд, Р.А. Минлос, З.Я. Шапиро. Представления группы вращений и группы Лоренца, их применения. М.: Физматгиз, 1958.

[5] L. M. Slad. Mod. Phys. Lett. A. 2000. V. 15. P. 379.

[6] M. Gell-Mann, M. Levy. Nuovo Cimento. 1960. V. 16. P. 705

[7] S. Coleman, J. Mandula. Phys. Rev. 1967. V. 159. P. 1251.

[8] Y. Nambu. Suppl. Progr. Theor. Phys. 1966. V. 37-38. P. 368; A. O. Barut, H. Kleinert. Phys. Rev. 1967. V. 157. P. 1180; C. Fronsdal. Phys. Rev. 1968. V. 171. P. 1811; G. Bisiacchi, P. Budini, G. Calucci. Phys. Rev. 1968. V. 172. P. 1508; H. Н. Боголюбов, А. А. Логунов, А. И. Оксак, И. Т. Тодоров. Общие принципы квантовой теории поля. М.: Наука, 1987.

[9] В. Л. Гинзбург, И. Е. Тамм. ЖЭТФ. 1947. Т. 17. С. 227.

[10] H. Yukawa. Phys. Rev. 1950. V. 77. P. 219.

[11] Ю. М. Широков. ЖЭТФ. 1951. Т. 21. С. 748.

[12] М. А. Марков. ДАН. 1955. Т. 101. С. 51.

[13] А.А. Комар, Л. М. Сладь. ТМФ. 1969. Т. 1. С. 50.

[14] S. Ström. Arkiv Fysik. 1965. V. 29. P. 467.

[15] K. Hagiwara et al. (Particle Data Group). Phys. Rev. D. 2002. V. 66. P. 010001.

[16] R. E. Cutkosky, S. Wang. Phys. Rev. D. 1990. V. 42. P. 235.

Поступила в редакцию 13.IV.2004 г. 